\title{
Liquid Biopsy in Metastatic Breast Cancer: Current Role of Circulating Tumor Cells and Circulating Tumor DNA
}

\author{
Maggie Banys-Paluchowskia, ${ }^{\text {banja N. Fehm }}{ }^{c}$ Donata Grimm-Glang ${ }^{a}$ \\ Achim Rody ${ }^{a}$ Natalia Krawczyk ${ }^{c}$ \\ ${ }^{a}$ Department of Obstetrics and Gynecology, University Hospital of Schleswig Holstein, Campus Lübeck,

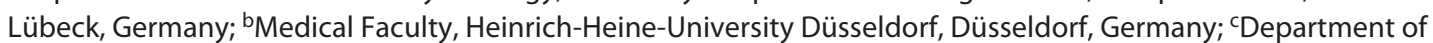 \\ Gynecology and Obstetrics, Heinrich-Heine-University Düsseldorf, Düsseldorf, Germany
}

\section{Keywords}

Metastatic breast cancer - Circulating tumor cell . Circulating tumor DNA · Liquid biopsy · Therapy interventions

\begin{abstract}
Background: In metastatic breast cancer (MBC), bloodbased diagnostics have become a major focus of oncological research in the last 2 decades. Detection of circulating tumor cells (CTCs) and circulating tumor DNA (ctDNA) has the potential to improve prognosis assessment and complement standard therapy monitoring tools. Summary: To date, several large analyses have confirmed high CTC counts as an independent prognostic factor. Persistently high CTC numbers during systemic treatment are associated with early progression, but it remains to be clarified which therapeutic options should be offered to such patients since the SWOG 0500 trial failed to show benefit from early switch to another chemotherapy regimen in patients with CTC persistence. In comparison, evidence on the prognostic value of ctDNA is still limited. Most importantly, liquid biopsy-guided treatment interventions have been investigated in several trials. In patients with hormone receptor-positive and HER2-negative MBC, CTC-driven therapy choices resulted in similar PFS to physician's choice treatment. Recently, the DETECT III trial has shown that patients with HER2-negative MBC and HER2positive CTCs may benefit from targeted anti-HER2 treatment with lapatinib. ctDNA-driven therapy selection has already been approved in clinical routine: alpelisib is the first
\end{abstract}

karger@karger.com www.karger.com/ort

Karger" BOPEN ACCESS
(C) 2021 The Author(s)

Published by S. Karger AG, Basel

This article is licensed under the Creative Commons Attribution 4.0 International License (CC BY) (http://www.karger.com/Services/ OpenAccessLicense). Usage, derivative works and distribution are permitted provided that proper credit is given to the author and the original publisher. targeted treatment indicated on the basis of a ctDNA test. Key Messages: CTCs and ctDNA predict clinical outcome and have a potential to improve therapy choices in MBC.

\section{(C) 2021 The Author(s).}

Published by S. Karger AG, Basel

\section{Introduction}

Hematogenous spread of single cancer cells in patients with breast cancer is a well-known phenomenon. While large quantities of these cells continuously leave the primary tumor, the vast majority is not able to survive in the circulation and only a small proportion has a chance to persist in secondary homing sites [1-4]. In case of a small tumor weighing $1 \mathrm{~g}$, an estimated $3 \times 10^{6}$ tumor cells enter blood circulation daily [4]. Despite these high numbers, the half-life of tumor cells leaving the primary tumor is somewhere between 1 and $2.4 \mathrm{~h}$, and this fact is often described as "metastatic inefficiency" [1]. Depending on the compartment the cells are detected in, they are referred to either as circulating tumor cells (CTCs; peripheral blood) or as disseminated tumor cells (other compartments such as bone marrow).

In the recent decades, CTCs have been established as a strong prognostic factor in metastatic breast cancer (MBC) and first studies have shown their potential to guide treatment decisions $[2,5,6]$. Beyond tumor cellbased analyses, circulating nucleic acids, such as DNA and RNA fragments, can also be routinely detected in peripheral blood [7]. They are released to the bloodstream 
by primary tumor, metastatic lesions, and decaying CTCs, and detection is usually carried out in blood plasma [7]. While the evidence on the prognostic value of circulating tumor DNA (ctDNA) detection in MBC is limited, particularly compared with level I evidence of CTCs, the possibility to detect somatic mutations via liquid biopsy appears promising and the first liquid biopsy-based therapy indication using ctDNA has recently been approved in the USA and European Union [7]. In the present review, we discuss the current evidence on the clinical role and potential future applications of CTC- and ctDNA-based diagnostics in MBC.

\section{Circulating Tumor Cells}

In early breast cancer, CTCs can be detected in a large proportion of patients and are widely assumed to represent a surrogate marker of minimal residual disease as well as potential precursors of subsequent metastatic disease. In a metastatic disease, these cells may complement diagnostic possibilities based on the hypothesis that they reflect both the tumor burden and the currently dominating cell populations of metastatic sites. One of the major advantages of the CTC-based diagnostics is the ability to perform serial measurements in a noninvasive manner, offering a "real-time" insight into the biology of the disease and potentially improving therapy selection. Since predictive markers, such as hormone and HER2 receptor status and new therapeutically relevant and targetable alterations (e.g., PIK3CA, AKT1, or ESR1 mutations), may change in the course of disease, liquid biopsy has a potential to become an alternative to invasive tissue biopsies in metastatic situation.

\section{Prognostic Relevance of CTCs}

In MBC, the presence of CTCs has been shown to be a strong independent prognostic factor. In contrast to early disease setting, where the cutoff level of 1 CTC is usually used for differentiation between patients with favorable and unfavorable outcomes, 5 CTCs per $7.5 \mathrm{~mL}$ of blood (measured by the CellSearch system) are frequently used for prognostication in metastatic disease [8-11]. In some studies, patients with $\geq 5$ CTCs were referred to as $\mathrm{CTC}_{\text {aggressive }}$ or $\mathrm{CTC}_{\text {high }}$ and those with $<5$ CTCs as $\mathrm{CTC}_{\text {indolent }}$ or $\mathrm{CTC}_{\text {low }}[6,8]$. Two large analyses on the prognostic value of CTCs have been recently published. In the pooled analysis conducted by Cristofanilli et al. [8], blood samples from nearly 2,500 MBC patients from 18 centers were evaluated, among them 533 women with de novo metastatic cancer. The CellSearch system was used for CTC detection. Elevated CTC numbers predicted significantly worse prognosis (median overall survival [OS]: 36.3 in $\mathrm{CTC}_{\text {aggressive }}$ vs. 16 months in $\mathrm{CTC}_{\text {indolent }}[p<$
0.0001]) [8] (online suppl. Table 1; for all online suppl. material, see www.karger.com/doi/10.1159/000520561), and this association was independent of the metastasis localization (OS in patients with visceral metastases: 13.2 vs. 29.9 months; in case of bone-only disease: 23.8 vs. 46.9 months, respectively) and tumor subtype. In MBC patients with de novo metastatic disease, elevated CTC counts were also associated with shorter OS (18.7 vs. 41.4 months, $p<0.0001)$. Importantly, the CTC status was the strongest $\mathrm{OS}$ predictor in the multivariate analysis (HR 2.71, 95\% CI: $2.35-3.12, p<0.0001$ ).

The second analysis was reported by the DETECT study group at the San Antonio Breast Cancer Symposium in 2020 [11]; the full publication is pending. Here, blood samples from $1933 \mathrm{MBC}$ patients screened for participation in DETECT III and IV trials were analyzed. The DETECT trials are the largest study program on CTCbased therapy interventions worldwide and were initiated to address the role of CTC characterization for the use of targeted therapy [12]. Specifically, patients with HER2negative disease and HER2-positive CTCs were randomized to receive standard treatment \pm anti-HER2 therapy with lapatinib in the DETECT III trial; those with HER2negative CTCs were invited to participate in DETECT IVa and IVb studies. The analysis of patients screened for these trials confirmed high prognostic relevance of CTC counts in metastatic disease [11]. Interestingly, both the 1 CTC and 5 CTCs cutoffs were significantly associated with OS (median OS in patients with $\geq 1$ CTC: 15.5 months [95\% CI 14.2-16.8] vs. 37.2 months in CTC-negative patients [95\% CI 32.7-41.7] and 12.0 months with $\geq 5$ CTCs [95\% CI 10.0-14.0] vs. 28.6 months with <5 CTCs [95\% CI 25.5-31.6]). In addition, the prognostic role of the HER2 status of CTCs was evaluated. In the univariate analysis, the presence of at least 1 CTC with strong HER2 staining was associated with worse OS (median OS: 9.7 [95\% CI 7.1-12.3] vs. 16.5 months in patients with HER2negative CTCs only [14.9-18.1], respectively, $p=0.013$, hazard ratio 1.360), but the difference was not significant in the multivariate analysis. This study remains the largest analysis conducted in HER2-negative MBC.

\section{CTCs for Therapy Monitoring}

Since most patients show a decrease in CTC counts during systemic treatment, detection of persistent CTCs can potentially contribute to correct identification of those with insufficient response to therapy $[13,14]$. Hypothetically, these patients could be spared inefficient but potentially toxic therapies or be switched to other treatment options.

This issue has been addressed by the SWOG-S0500 (NCT00382018) trial [14]. In this study, CTCs were measured in $595 \mathrm{MBC}$ patients prior to begin of first-line cytotoxic therapy. A second blood sample was obtained after 
the first cycle. Patients with persistently high CTC counts were randomized to continuation of therapy until radiological and/or clinical progression (standard arm) or switched to another chemotherapy regimen (CTC-driven arm). While patients with initially low CTC numbers had most favorable clinical outcomes ( 35 months median OS), the prognosis was similar in women with persistently high CTC numbers in both arms (13 months median OS). Therefore, switching therapy to another regimen based on early detection of insufficient CTC response did not improve prognosis. Possibly, CTC persistence indicates a general resistance to conventional chemotherapies, and patients might be more likely to benefit from immunologic, targeted, or experimental therapy options.

Another study investigating CTC-based therapy monitoring was the CirCe01 trial (NCT01349842) [15]. In this randomized phase III study, patients scheduled to begin their $\geq 3$ rd line of therapy were randomized between the standard arm and the CTC-driven arm. In the CTC-driven am, CTC dynamics were used to guide an early switch to another line of therapy. The study did not reach its primary endpoint: survival was similar in both arms (HR $0.95, p=0.8$ ). However, compliance with the proposed early switch of chemotherapy in patients with no CTC response nevertheless resulted in quantitatively longer median PFS and OS.

\section{CTC-Driven Selection of Therapy}

The ultimate "holy Grail" of liquid biopsy-based research is development of treatment interventions guided by blood sampling. In MBC, therapy choices are so far based on histological characteristics of the disease, evaluated using metastatic or primary tumor tissue. However, predictive markers, such as hormone receptor and HER2 status, may change during the course of a disease (online suppl. Table 2, [16]), and therefore, current guidelines recommend a reevaluation of these markers using a metastatic biopsy [17-19]. Despite this recommendation, a biopsy of metastatic site(s) is not feasible in many patients, for example, in case of an untypical localization of metastasis or patient's general condition. In these patients, therapy choices are based on tissue characteristics assessed many years ago. Therefore, attempts have been made to establish the role of CTC examination to guide treatment in the metastatic setting.

The first positive study on CTC-driven treatment choices was the French STIC CTC trial $[6,20]$. This phase III noninferiority study was designed to treat $778 \mathrm{MBC}$ patients with hormone receptor-positive HER2-negative disease who were randomized to physician's choice therapy or CTC-driven treatment (i.e., endocrine monotherapy or chemotherapy) as their first-line treatment. Peripheral blood was evaluated prior to begin of treatment in all study participants using the CellSearch assay. 27\% of patients had high CTC counts (i.e., $\geq 5$ CTCs $/ 7.5 \mathrm{~mL}$ ). In the physician's choice arm, patients were treated by either chemotherapy or endocrine monotherapy at the discretion of patient's oncologist. In the CTC-driven arm, study participants with $\geq 5$ CTCs were treated with chemotherapy, and patients with $<5$ CTCs received endocrine therapy. $73 \%$ of patients in the physician's choice arm received endocrine therapy, compared to $63 \%$ in the CTC-driven arm [6].

The STIC CTC study showed similar PFS in both arms, and therefore the noninferiority of CTC-driven treatment choice, as compared to physician's choice of therapy (median PFS: 13.9 months [95\% CI, 12.2-16.3] in the physician's choice arm and 15.5 months [95\% CI, 12.717.3] in the CTC-driven arm). In patients with a discordant treatment recommendation (i.e., clinically low-risk but CTC-high and clinically high-risk but CTC-low), PFS and OS were longer in study participants receiving chemotherapy than endocrine therapy, showing that these patients benefit from CTC diagnostics. It remains difficult to place these results within the current therapy landscape, particularly because CDK4/6 inhibitors, now considered standard of care in the first-line setting, have been approved after the trial begun and were therefore not allowed in either arm.

Another positive study on the CTC-driven interventions was the aforementioned DETECT III trial [5], which randomized 105 tissue HER2-negative MBC patients harboring HER2-positive CTCs to either standard treatment alone or standard treatment in combination with lapatinib (NCT01619111). The CTC clearance rate at the end of study treatment (mostly at time of progression) was similar in both arms (standard arm: 33\%, compared to $15 \%$ in lapatinib arm, $p=0.18$ ). The OS was significantly longer in the lapatinib arm (univariable analysis: HR 0.54, $p=0.008$; multivariable analysis: $\mathrm{HR} 0.55, p=0.016)$. PFS was numerically longer in the lapatinib arm $(\operatorname{HR} 0.69, p=$ $0.14)$. The full publication of these results is still awaited.

\section{Circulating Tumor DNA}

$\mathrm{ctDNA}$ is tumor-specific free DNA fragments released from the primary tumor, metastatic lesions, or isolated tumor cells mostly during the process of necrosis or apoptosis. Based on tumor-specific mutations, the ctDNA can be differentiated from other nonspecific free DNA fragments detected in peripheral blood, which are usually referred to as cell-free DNA (cfDNA) or free circulating DNA and originate from normal cells.

In general, ctDNA is isolated from blood plasma and its burden refers to the relative amount of ctDNA in comparison to the total amount of cfDNA, which can be very small. Therefore, highly sensitive techniques are required
Oncol Res Treat 2022:45:4-10

DOI: $10.1159 / 000520561$
Banys-Paluchowski/Fehm/Grimm-Glang/ Rody/Krawczyk 
for ctDNA detection. Currently, 2 main techniques are used for ctDNA detection: targeted and untargeted approach. The targeted approach, for example, digital droplet PCR or beads amplification magnetics PCR (BEAMing-PCR), is used to detect previously determined tumorspecific mutations in genes such as PIK3CA, AKT1, ESR1, $\mathrm{PTEN}$, or ERB2. The term digital next-generation sequencing, on the other hand, describes several untargeted approaches, for example, whole-genome sequencing, exome sequencing, or array CGH (array comparative genomic hybridization) that allow sequencing of large DNA or RNA molecules and are able to detect previously unknown genetic alternations [21].

\section{Prognostic Relevance of ctDNA}

While the prognostic significance of CTC in breast cancer has been demonstrated in several studies and confirmed by large meta-analyses, there is limited evidence on the prognostic value of ctDNA in breast cancer (online suppl. Table 3). A meta-analysis of 10 trials published by Tan et al. in 2018 included overall only 1,127 patients, mostly with nonmektastatic disease [22]. To date, only a few studies evaluated the prognostic significance of $\mathrm{ctD}$ NA in metastatic setting. Shaw et al. [23] compared mutation profiles in CTCs and ctDNA in $112 \mathrm{MBC}$ patients. Elevated ctDNA levels, identified by detection of mutations in PIK3CA, KRAS, TP53, and ESR1 genes, were associated with significantly shorter OS and mutation profiles in ctDNA and CTCs were concordant [23]. In the study by Fernandez-Garcia et al. [24], blood samples from $194 \mathrm{MBC}$ patients were analyzed for CTC counts, cfDNA levels, and tumor markers CA15-3 and AP. High levels of cfDNA were associated with shorter OS ( $p$ value $<0.0001, \mathrm{HR}=2.296, \mathrm{CI}=1.476-3.570)$ and overall cfDNA levels correlated with CTC counts [24]. Pierga et al. [25] recently reported the results of the prospective $U C B G$ COMET trial that included 196 HER2-negative MBC patients analyzed for ctDNA levels at baseline and before second cycle of chemotherapy. Several somatic mutations, that is, TP53, PIK3CA, ESR1, and HER2, have been tested by targeted resequencing. Interestingly, a detection of TP53 mutation in plasma at baseline was found to affect PFS and OS, whereas PIK3CA or ESR1 mutations had no impact on survival in these patients. Moreover, patients with detectable ctDNA before second cycle of chemotherapy had significantly shorter OS. In summary, there is growing evidence, suggesting a prognostic impact of ctDNA in MBC. However, the direct comparison of the published trials is difficult because of heterogeneous methodology.

\section{ctDNA for Therapy Monitoring}

Several clinical trials integrated ctDNA analysis in order to evaluate its utility as a therapy monitoring tool in
MBC patients (online suppl. Table 4). In the phase III PALOMA III trial, $521 \mathrm{HR}$-positive patients with disease progression under endocrine treatment were randomized to combination therapy with fulvestrant and palbociclib versus fulvestrant and placebo [26]. ctDNA testing for hotspot mutations in PIK3CA and ESR1 genes using multiple digital PCR was performed at baseline and day 15 of the first treatment cycle. Of 455 blood samples available at baseline, at least 1 PIK3CA hotspot mutation was detected in 100 patients (22\%). Matched blood samples from 73 of these patients were also analyzed on day 15 . Significantly lower levels of PIK3CA ctDNA were detected at day 15 of therapy in palbociclib arm comparing to placebo arm. Moreover, the drop of PIK3CA ctDNA strongly predicted PFS in patients treated with palbociclib + fulvestrant (HR 3,94, 95\% CI 1.61-9.64, long rank $p=0.0013)$. Interestingly, although ESR1 copies at day 15 were also significant lower in palbociclib arm than in placebo arm $(p=0.0034)$, ESR1 ctDNA dynamics did not predict clinical outcome of patients in this analysis (HR $1.68,95 \%$ CI $0.74-3.82, p=0.21$ ).

The predictive value of early ctDNA changes was confirmed in the phase II BEECH trial, which included patients with ER + MBC treated with paclitaxel + AKT inhibitor capivasertib versus placebo [27]. In this analysis, an untargeted ctDNA approach was performed at baseline to identify mutations for serial testing and second several specific mutations (i.e., AKT1, PIK3CA, ATM, TP53, ERB2, ESR1, etc.) have been tested by digital droplet PCR to assess ctDNA changes during the treatment. A drop in ctDNA at 4 weeks was shown to predict PFS in these studies (11.1 vs. 6.4 months, $\mathrm{HR}=0.20,95 \% \mathrm{CI}$ $0.083-0.50, p<0.0001)$. Moreover, ctDNA suppression was similar in both study arms, thus reflecting the lack of clinical benefit of treatment with capivasertib demonstrated in this trial [28].

A pooled analysis of ctDNA evaluation performed in MONALEESA 2-, 3- and 7-trials was presented at the ASCO Symposium 2020 [29]. Blood samples from 1,507 ER + HER2-negative MBC patients were analyzed at baseline by the NGS targeted panel for alterations in 557 genes. Several gene changes detected in the plasma of those patients demonstrated a predictive value for therapy with ribociclib. Changes in FRS2, PRKCA, MDM2, ERB2, AKT1, and BRCA1/2 predicted a trend for increased PFS benefit of ribociclib treatment, while there was only little or no benefit from ribociclib in patients with alterations in CHD4, BCL11B, ATM, or $\mathrm{CDKN} 2 \mathrm{~A} / 2 \mathrm{~B} / 2 \mathrm{C}$ genes. Another trial that evaluated the predictive value of ctDNA in MBC patients was a large phase III PADA1 study. This trial analyzed the clinical impact of ESR1 mutation in the blood plasma of 1,017 ER-positive HER2-negative patients treated with firstline aromatase inhibitors and palbociclib [30]. According
Oncol Res Treat 2022;45:4-10

DOI: $10.1159 / 000520561$
Banys-Paluchowski/Fehm/Grimm-Glang/ Rody/Krawczyk 
to early results also presented at the ASCO Symposium 2020, ESR1 mutations detected in ctDNA predicted a significantly shorter PFS on treatment with aromatase inhibitors + palbociclib comparing to patients with wildtype ESR1 detected in plasma (11.0 vs. 26.7 months, HR $=2.3$ ). Further, patients with ESR1 mutations without progress under study treatment were then randomized to continue the same therapy or switch to fulvestrant and palbociclib. These results have not been reported to date. Therefore, it remains to be seen if these and further results of PADA1 trial will affect therapeutic decisions in patients with ctDNA detected ESR1 mutation in everyday clinical practice.

\section{ctDNA-Driven Selection of Therapy}

While the prognostic and predictive impact of ctDNA in $\mathrm{MBC}$ have been less intensively investigated than in case of CTCs, ctDNA is widely assumed to hold a great potential for specific drug matching by identifying known or new "targetable" mutations in BC patients (online suppl. Table 5). In this context, the approval of the PI3K-inhibitor alpelisib by FDA in 2019 and EMA (European Medicines Agency) in 2020 for PIK3CA-mutated MBC represents the first ctDNA-based indication in $\mathrm{BC}$ treatment [31]. Alpelisib targets the PI3K/Akt/Mtor signaling pathway that is crucial for tumor cell growth and proliferation and is activated in patients with hot spot PIK3CA mutations, seen in up to $40 \%$ of advanced BC cases. In the randomized phase III SOLAR 1 trial, a total of $572 \mathrm{ER}+$ HER2-endocrine-pretreated MBC patients were randomized to therapy with fulvestrant + alpelisib versus fulvestrant + placebo. PIK3CA mutation status was assessed in tumor tissue in all 572 patients and in plasma in 381. Treatment with alpelisib was associated with significant PFS benefit in PIK3CA-mutated patients (11 vs. 5.7 months, HR $0.65,95 \%$ CI $0.50-0.85, p<0.001$ ). This effect could be observed regardless of whether the PIK3CA mutation was detected in tumor tissue or in ctDNA. The recently published final data analysis of this trial has shown a numerical but not significant OS benefit of 7.9 months for alpelisib in PIK3CA-mutated group (HR 0.86 , 95\% CI 0.64-1.15, $p=0.15$ ) [32]. Interestingly, patients with PIK3CA mutation in ctDNA demonstrated higher, but still not significant OS benefit from alpelisib treatment in this trial (HR 0.74, 95\% CI 0.51-1.08).

Another study investigating ctDNA-guided treatment of targetable mutations in MBC patients was the plasmaMATCH trial [33]. In this phase IIa study, ctDNA samples from 1,034 patients were tested for specific mutations, and patients were then divided into 4 cohorts, based on the result. Patients with ESR1 mutations were treated with the extended dose of fulvestrant (cohort A); patients with HER 2 mutations were treated with oral neratinib; and in case of ER-positivity with a standard dose of ful- vestrant (cohort B), ER-positive patients with AKT1-mutations received capivasertib + standard dose of fulvestrant (cohort C) and ER-negative patients with AKT1 mutation or patients with PTEN mutations received capivasertib alone. The primary endpoint was defined as the objective response rate for each cohort and has been reached in cohorts B and C with $25 \%$ and $22 \%$ response rates for neratinib and capivasertib, respectively.

\section{Conclusions}

- The prognostic value of CTC detection in patients with $\mathrm{MBC}$ is very high (cutoff of 5 cells $/ 7.5 \mathrm{~mL}$ peripheral blood).

- Dynamics of CTCs and ctDNA can predict response to systemic treatment in MBC.

- ctDNA analysis allows the noninvasive identification of potentially targetable hot spot mutations in MBC.

- Liquid biopsy-based therapeutic interventions are in focus of currently ongoing oncological MBC trials.

- The PI3K-inhibitor alpelisib is the first liquid biopsybased approved agent in BC therapy.

\section{Conflict of Interest Statement}

Maggie Banys-Paluchowski received honoraria for lectures and advisory role from Novartis, Roche, Pfizer, Eli Lilly, Eisai, Daiichi Sankyo, GSK, AstraZeneca, pfm, and Amgen and study support from Endomag, Mammotome, and MeritMedical. Other authors report no conflicts of interest.

\section{Funding Sources}

No funding was received to support writing of the manuscript.

\section{Author Contributions}

M.B.P., N.K., and T.F. wrote and edited the manuscript. A.R. and D.G.: edited the manuscript.

\section{References}

\footnotetext{
1 Meng S, Tripathy D, Frenkel EP, Shete S, Naftalis EZ, Huth JF, et al. Circulating tumor cells in patients with breast cancer dormancy. Clin Cancer Res. 2004;10(24):8152-62.

2 Banys-Paluchowski M, Reinhardt F, Fehm T. Circulating tumor cells in metastatic breast cancer: clinical applications and future possibilities. Appl Sci. 2020;10(9):3311.

3 Krawczyk N, Hartkopf A, Banys M, MeierStiegen F, Staebler A, Wallwiener M, et al. Prognostic relevance of induced and spontaneous apoptosis of disseminated tumor cells in primary breast cancer patients. BMC Cancer. 2014;14:394.
} 
4 Butler TP, Gullino PM. Quantitation of cell shedding into efferent blood of mammary adenocarcinoma. Cancer Res. 1975;35(3):5126.

5 Fehm T, Müller V, Banys-Paluchowski M, fasching PA, Friedl TWP, Hartkopf A, et al. Efficacy of the tyrosine kinase inhibitor lapatinib in the treatment of patients with HER2negative metastatic breast cancer and HER2positive circulating tumor cells: results from the randomized phase III DETECT III trial. Canc Res. 2020.

6 Bidard FC, Jacot W, Kiavue N, Dureau S, Kadi A, Brain E, et al. Efficacy of circulating tumor cell count-driven versus clinician-driven first-line therapy choice in hormone receptor-positive, ERBB2-negative metastatic breast cancer: the STIC CTC randomized clinical trial. JAMA Oncol. 2021;7(1):34-41.

7 Banys-Paluchowski M, Krawczyk N, Fehm T. Liquid biopsy in breast cancer. Geburtshilfe Frauenheilkd. 2020;80(11):1093-104.

8 Cristofanilli M, Pierga JY, Reuben J, Rademaker A, Davis AA, Peeters DJ, et al. The clinical use of circulating tumor cells (CTCs) enumeration for staging of metastatic breast cancer (MBC): International expert consensus paper. Crit Rev Oncol Hematol. 2019;134: 39-45.

9 Bidard FC, Peeters DJ, Fehm T, Nolé F, Gisbert-Criado R, Mavroudis D, et al. Clinical validity of circulating tumour cells in patients with metastatic breast cancer: a pooled analysis of individual patient data. Lancet Oncol. 2014;15(4):406-14.

10 Banys-Paluchowski M, Krawczyk N, Fehm T. Potential role of circulating tumor cell detection and monitoring in breast cancer: a review of current evidence. Front Oncol. 2016;6:255.

11 Müller V, Banys-Paluchowski M, Fried TWP, Fasching PA, Schneeweiss A, Hartkopf A, et al. Prognostic relevance of the HER2 status of circulating tumor cells in metastatic breast cancer patients screened for participation in the DETECT study program. Can Res. 2020

12 Schramm A, Friedl TW, Schochter F, Scholz C, de Gregorio N, Huober J, et al. Therapeutic intervention based on circulating tumor cell phenotype in metastatic breast cancer: concept of the DETECT study program. Arch Gynecol Obstet. 2016;293(2):271-81.

13 Martin M, Custodio S, de Las Casas ML, Garcia-Saenz JA, de la Torre JC, Bellon-Cano JM, et al. Circulating tumor cells following first chemotherapy cycle: an early and strong predictor of outcome in patients with metastatic breast cancer. Oncologist. 2013;18(8):917-23.
14 Smerage JB, Barlow WE, Hortobagyi GN, Winer EP, Leyland-Jones B, Srkalovic G, et al. Circulating tumor cells and response to chemotherapy in metastatic breast cancer: SWOG S0500. J Clin Oncol. 2014;32(31): 3483-9.

15 Cabel L, Berger F, Cottu P, Loirat D, Rampanou A, Brain E, et al. Clinical utility of circulating tumour cell-based monitoring of lateline chemotherapy for metastatic breast cancer: the randomised CirCe01 trial. $\mathrm{Br} \mathrm{J}$ Cancer. 2021.

16 Schrijver WAME, Suijkerbuijk KPM, van Gils $\mathrm{CH}$, van der Wall E, Moelans CB, van Diest PJ. Receptor conversion in distant breast cancer metastases: a systematic review and metaanalysis. J Natl Cancer Inst. 2018;110(6):56880.

17 Recommendations of the AGO Breast Committee. diagnosis and treatment of patients with early and advanced breast cancer; 2021. Available from: www.ago-online.de.

18 NCCN Clinical Practice Guidelines in Oncology. Breast cancer; 2021. Available from: NCCN.org.

19 Ditsch N, Untch M, Kolberg-Liedtke C, Jackisch C, Krug D, Friedrich M, et al. AGO recommendations for the diagnosis and treatment of patients with locally advanced and metastatic breast cancer: update 2020. Breast Care. 2020;15(3):294-309.

20 Bidard FC, Jacot W, SD, Brain E, Bachelot T, $\mathrm{HB}$, et al. Abstract GS3-07: clinical utility of circulating tumor cell count as a tool to chose between first line hormone therapy and chemotherapy for ER + HER2 metastatic breast cancer: results of the phase III STIC CTC trial. Cancer Res. 2019.

21 Alix-Panabieres C, Pantel K. Clinical applications of circulating tumor cells and circulating tumor DNA as liquid biopsy. Cancer Discov. 2016;6(5):479-91.

22 Tan G, Chu C, Gui X, Li J, Chen Q. The prognostic value of circulating cell-free DNA in breast cancer: A meta-analysis. Medicine (Baltimore). 2018;97(13):e0197. http:dx.doi. org/10.1097/MD.0000000000010197.

23 Shaw JA, Guttery DS, Hills A, FernandezGarcia D, Page K, Rosales BM, et al. Mutation analysis of cell-free DNA and single circulating tumor cells in metastatic breast cancer patients with high circulating tumor cell counts. Clin Cancer Res. 2017;23(1):88-96.

24 Fernandez-Garcia D, Hills A, Page K, Hastings RK, Toghill B, Goddard KS, et al. Plasma cell-free DNA (cfDNA) as a predictive and prognostic marker in patients with metastatic breast cancer. Breast Cancer Res. 2019;21(1): 149.
25 Pierga J-Y, Silveira A, Girard E, Lorgis V, Tanguy M-L, Albaud B, et al. Abstract 3390: predictive and prognostic value of circulating tumor DNA (ctDNA) compared to circulating tumor cells (CTC) in a prospective cohort of metastatic breast cancer patients: the UCBG COMET trial. Cancer Res. 2020; 80(16):3390.

26 O'Leary B, Hrebien S, Morden JP, Beaney M, Fribbens C, Huang X, et al. Early circulating tumor DNA dynamics and clonal selection with palbociclib and fulvestrant for breast cancer. Nat Commun. 2018;9(1):896.

27 Hrebien S, Citi V, Garcia-Murillas I, Cutts R, Fenwick K, Kozarewa I, et al. Early ctDNA dynamics as a surrogate for progression-free survival in advanced breast cancer in the BEECH trial. Ann Oncol. 2019;30(6):945-52.

28 Turner NC, Alarcón E, Armstrong AC, Philco M, López Chuken YA, Sablin MP, et al. BEECH: a dose-finding run-in followed by a randomised phase II study assessing the efficacy of AKT inhibitor capivasertib (AZD5363) combined with paclitaxel in patients with estrogen receptor-positive advanced or metastatic breast cancer, and in a PIK3CA mutant sub-population. Ann Oncol. 2019;30(5):77480.

29 Andre F, Su F, Solovieff N, Arteaga CL, Hortobagyi GN, Chia SKL, et al. Pooled ctDNA analysis of the MONALEESA (ML) phase III advanced breast cancer (ABC) trials. J Clin Oncol. 2020;38(15):1009.

30 Bidard FC, Callens C, Dalenc F, Pistilli B, De La Motte Rouge T, Clatot F, et al. Prognostic impact of ESR1 mutations in ER + HER2MBC patients prior treated with first line AI and palbociclib: an exploratory analysis of the PADA-1 trial. J Clin Oncol. 2020:38.

31 Andre F, Ciruelos E, Rubovszky G, Campone M, Loibl S, Rugo HS, et al. Alpelisib for PIK3CA-mutated, hormone receptor-positive advanced breast cancer. N Engl J Med. 2019; 380(20):1929-40.

32 Andre F, Ciruelos EM, Juric D, Loibl S, Campone M, Mayer IA, et al. Alpelisib plus fulvestrant for PIK3CA-mutated, hormone receptor-positive, human epidermal growth factor receptor-2-negative advanced breast cancer: final overall survival results from SOLAR-1. Ann Oncol. 2021;32(2):208-17.

33 Turner NC, Kingston B, Kilburn LS, Kernaghan S, Wardley AM, Macpherson IR, et al. Circulating tumour DNA analysis to direct therapy in advanced breast cancer (plasmaMATCH): a multicentre, multicohort, phase 2a, platform trial. Lancet Oncol. 2020;21(10): 1296-308. 